\title{
Quantified Magnetic Resonance Spectroscopy in the Diagnosis of Burning Mouth Syndrome
}

\author{
Duzgun Yildirim 1*, Deniz Alis², Ceren Alis ${ }^{3}$, Filiz Namdar Pekiner ${ }^{4}$, Alev Bakir ${ }^{5}$, Suha Turkmen ${ }^{6}$, \\ Dastan Temirbekov ${ }^{7}$
}

\footnotetext{
${ }^{1}$ Acibadem University, Vocational School of Health Sciences, Department of Medical Imaging, Istanbul, Turkey

${ }^{2}$ Cerrahpasa University, School of Medicine, Department of Radiology, Istanbul, Turkey

${ }^{3}$ Istanbul University, Cerrahpasa Faculty of Medicine, Department of Neurology, Istanbul, Turkey

${ }^{4}$ Marmara University, Department of Maxillofacial Surgery, Istanbul, Turkey

${ }^{5}$ Halic University, School of Medicine, Department of Biostatistics and Medical Informatics, Istanbul, Turkey

${ }^{6}$ Acibadem University, School of Medicine, Department of Emergency Medicine, Istanbul, Turkey

${ }^{7}$ Anatomica Hospital, Department of HNS, Istanbul, Turkey

Email: *yildirimduzgun@yahoo.com,drdenizalis@gmail.com, cerencivcik@gmail.com, fpekiner@gmail.com,

alefbakir@gmail.com, suha.turkmen@acibadem.com.tr, dasekeeee@gmail.com
}

How to cite this paper: Yildirim, D., Alis, D., Alis, C., Pekiner, F.N., Bakir, A., Turkmen, S. and Temirbekov, D. (2019) Quantified Magnetic Resonance Spectroscopy in the Diagnosis of Burning Mouth Syndrome. Open Journal of Medical Imaging, 9, 33-41.

https://doi.org/10.4236/ojmi.2019.93003

Received: July 19, 2019

Accepted: September 14, 2019

Published: September 17, 2019

Copyright $\odot 2019$ by author(s) and Scientific Research Publishing Inc. This work is licensed under the Creative Commons Attribution International License (CC BY 4.0).

http://creativecommons.org/licenses/by/4.0/

\section{(c) (i) Open Access}

\begin{abstract}
Purpose: To investigate potential pathological differences in major brain metabolites in burning mouth syndrome (BMS). Materials and Methods: We prospectively evaluated eight patients and six healthy controls with single voxel magnetic resonance (MR) spectroscopy throughout 2017 using 3-Tesla MR unit. Metabolite levels measured from the left posterior paracingulate white matter. Raw images were processed with Tarquin version 4.3.10 and area under curve values were automatically calculated for GABA ( $\gamma$-Aminobutyric acid), Ins (myo-inositole), tNAA (N-Acetylaspartate), tCho (total choline), $\mathrm{tCr}$ (total creatine) and GLx (glutamine + Glutamat). Major metabolite levels and all metabolites ratio to $\mathrm{tCr}$ calculated and compared between two groups using Mann-Whitney $U$ test. Results: GABA/tCR value of the patients $(0.589 \pm 0.194$, median $=0.515)$ was significantly higher than control group $(0.230 \pm 0.067$, median $=0.220)(\mathrm{P}=0.002)$. $\mathrm{tNAA} / \mathrm{tCR}$ value of the patients ( $1.106 \pm 0.218$, median: 1.205$)$, was significantly lower than the control group $(1.538 \pm 0.401$, median: 1.705$)(P=0.028)$. No significant difference was observed between two groups in terms of all other metabolites $(\mathrm{P}>0.05)$. Conclusion: We demonstrated increased GABA and decreased NAA in the left posterior paracingulate region in BMS patients. We suggest that these metabolite alterations in central nervous system might play a key role in the etiology of the disease by inhibiting the suppression of burning sensation.
\end{abstract}




\section{Keywords}

Burning Mouth Syndrome, BMS, GABA, MRI, Spectroscopy

\section{Introduction}

Burning mouth syndrome (BMS) refers to chronic orofacial pain without any mucosal lesions or other evident clinical signs. It is commonly observed in middle-aged patients and postmenopausal women [1]. BMS is mainly characterized by intense burning or stinging sensation in the oral mucosa [1]. Furthermore, it can also be accompanied by other sensory disorders such as dry mouth or taste alterations. Probably of multifactorial origin, and often idiopathic, with a still unknown etiopathogenesis in which local, systemic and psychological factors are implicated [2]. Currently, there is no consensus on the diagnosis and classification of BMS.

Although many hypotheses have been suggested on the etiology of the disease, alterations in the level of $\gamma$-Aminobutyric acid (GABA), a neurotransmitter that acts as an inhibitor in the brain, is widely accepted as a primary factor in the development of the disease [3] [4]. Recent studies indirectly demonstrated the presence of decreased GABA levels in BMS patients by showing improvement of symptoms in BMS patients under treatment that indirectly increased GABA levels [5] [6]. However, it has not yet been answered whether a lack of GABA in all brain or lack of GABA in certain pathways leading to BMS syndrome.

In our study, we quantitatively measured the level of GABA and other main metabolites, which would likely to demonstrate changes in case of neuronal dysfunction, including Ins (myo-inositole), tNAA (N-Acetylaspartate), tCho (total choline), $\mathrm{tCr}$ (total creatine) and GLx (glutamine + Glutamat), in the brain with magnetic resonance spectroscopy (MRS) to test these hypotheses and evaluate potential alterations in major brain metabolites that might give valuable information regarding BMS etiology.

\section{Materials and Methods}

Local ethics committee of our University approved this prospective study with an ethic number of 191,533 and informed consents were obtained from all the participants. We prospectively evaluated eight BMS patients, without any known systemic disease, malignancy or infection, (five female and three male) and six healthy controls (three female and three male) throughout 2017 with single voxel Magnetic resonance (MR) spectroscopy (TR: 2000 ms, TE: 30 ms, FA: 90 degree, Averages: 80) using 32-channel head coil with 48 channel 3-Tesla MR unit (MAGNETOM Skyra; Siemens Healthcare, Erlangen, Germany). Patients included in the study had previously been received medications for similar symptoms, at least for three years, but had not showed any improvement. These patients thoroughly evaluated by dedicated team consist of internal medicine spe- 
cialist, ear, nose, throat surgeon and experienced dental specialist. Their systemic and oral psychical examinations were performed, and their biochemical and blood parameters were extensively explored; yet yielded no systemic or local pathology. Therefore, these patients ( 8 patients, 5 female and 3 male, mean age 54) were diagnosed with primer BMS clinically, and included in the group 1, while age and sex-matched healthy participants (6 participants, 3 male and 3 female, mean age 48) were included in the group 2. During the study period, none of the patients or healthy controls was receiving any medications since that might have lead to inconclusive results. The cases that used psychiatric GABA related drugs were not included in the study. For the healthy group, the cases who had previously had BMS like symptoms or had mucosal discoloration, inflammation, or had dental restoration procedure were not included.

MRS is an MR technique, which enables to measure various metabolities in-vivo. Various metabolities in different regions of the brain could be analyzed depending on the relevant pathology. In our study, metabolite levels measured from the left posterior paracingulate white matter and were recorded separately for all participants using same parameters. Raw data and images regarding these metabolites (Figure 1(a)) were processed with Tarquin version 4.3.10 and area under curve values were automatically calculated for GABA ( $\gamma$-Aminobutyric acid), Ins (myo-inositole), tNAA (N-Acetylaspartate), tCho (total choline), $\mathrm{tCr}$ (total creatine) and GLx (glutamine + Glutamat) (Figure 1(b)).

In the following paragraph, we gave brief information concerning the role of the metabolites, which we measured, in the central nervous system to enhance the readability of the text and to better reflect the main purposes of the study. GABA is the primary inhibitory neurotransmitter in the central nervous system [7] [8]. Ins is a glial marker, which represents glial proliferation regardless of the underlying pathology [7] [8]. NAA associated with neuronal viability and it has very high levels in the normal healthy adult brain parenchyma [7] [8]. Cho is mainly located in the cell membrane, and increased levels are expected in case of inflammatory or neoplastic disorders, which disturbs cell membrane integrity [7] [8]. All metabolites that belong to Glx group have excitatory functions and enhance synaptic transmission [7] [8]. Cr represents energy storage, similarly with the heart and the skeletal muscles, and since it has relatively constant levels compared to others, it commonly used as a reference metabolites to detect slight changes in the others [7] [8].

Finally, the measured major metabolite levels were compared for both groups. All metabolites ratio (GABA, Ins, NAA, Cho, Glx) to $\mathrm{tCr}$ were also calculated and compared between two groups using Mann-Whitney $\mathrm{U}$ test (Table 1) $(\mathrm{P}<$ 0.05 was accepted as significant)

\section{Results}

$\mathrm{GABA} / \mathrm{tCR}$ value of the group $1(0.589 \pm 0.194$, median $=0.515)$ was significantly higher than group $2(0.230 \pm 0.067$, median $=0.220)(P=0.002) . \mathrm{tNAA} / \mathrm{tCR}$ value 


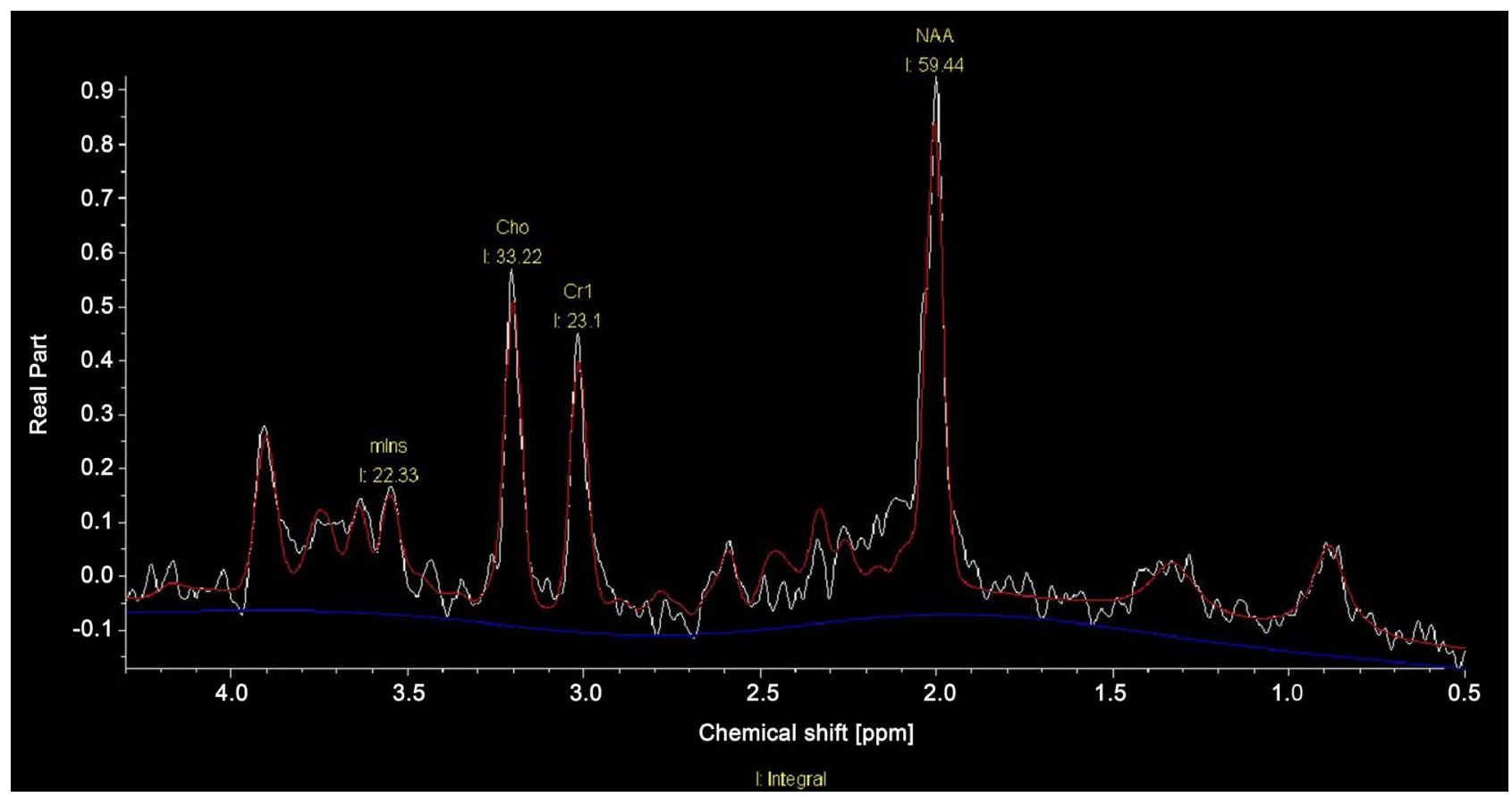

(a)
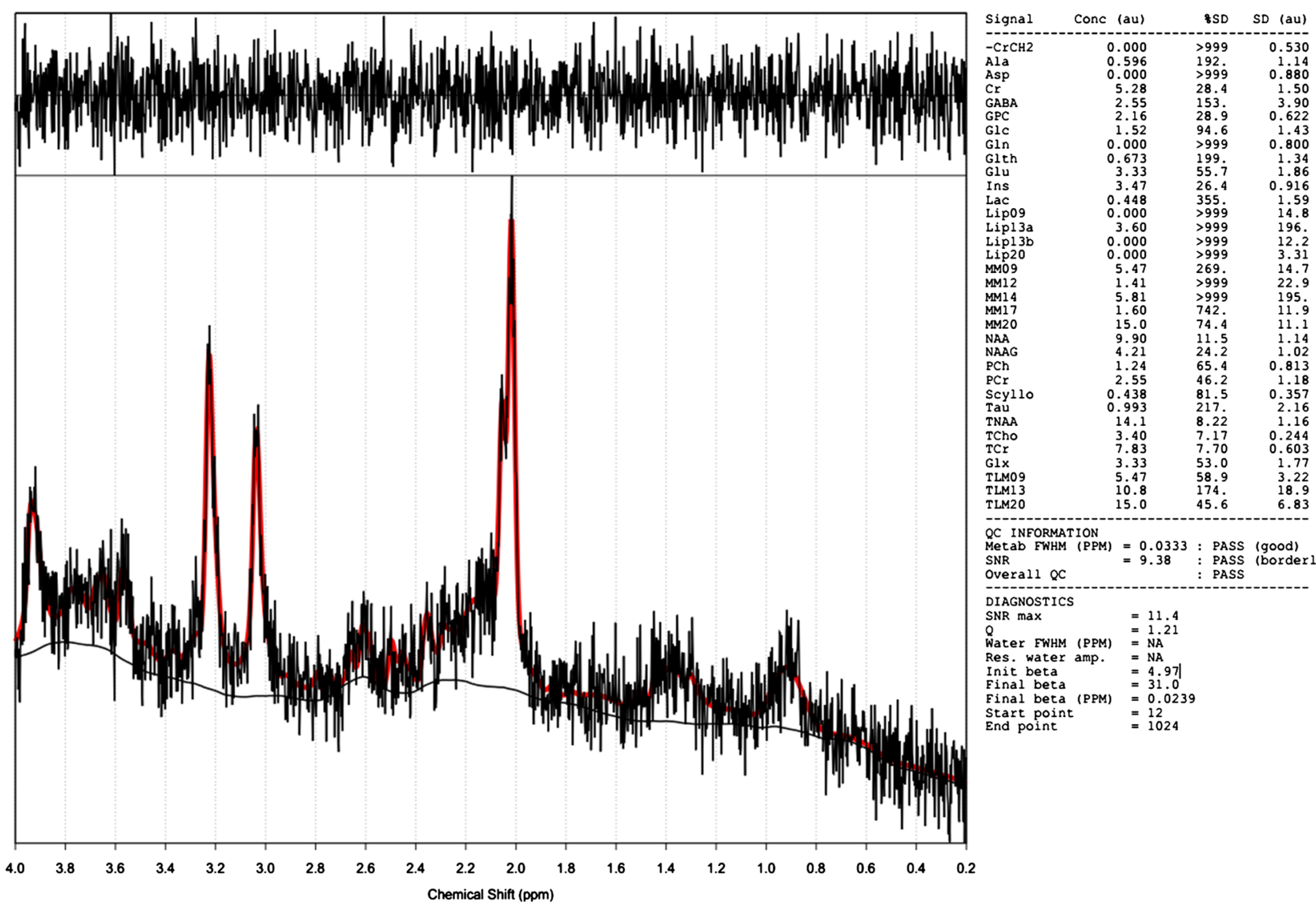

QC INFORMATION SWR Overall QC

DIAGNOSTICS

$\begin{array}{ll}\text { SNR } \max & =11.4 \\ Q & =1.21\end{array}$

Water FWHM (PPM) $=$ NA

Res. water a

$=4.97$
$=31.0$

Final beta (PPM) $=0.023$

$\begin{array}{ll}\text { Start point } & =12 \\ \text { End point } & =1024\end{array}$

(b)

Figure 1. The single voxel spectroscopy. Peak values of the metabolites in first automatic window view (a) and more detailed quantitative metabolite values that were processed in Tarquin software (b). GABA: $\gamma$-Aminobutyric acid, Ins: myo-inositole, tNAA: N-Acetylaspartate, tCho: total choline, tCr: total creatine, GLx: glutamine + Glutamat. 
of the group $1(1.106 \pm 0.218$, median $=1.205)$, was significantly lower than the group $2(1.538 \pm 0.401$, median $=1.705)(P=0.028<0.05)$. No significant difference was observed between two groups in terms of all other metabolites $(P>$ 0.05). Moreover, a mild increase in Ins and Glx levels were identified in BMS grouped compared to controls, yet without statistical significance $(P>0.05)$. Table 2 shows geographical data and metabolite levels of all the participants and Table 1 shows the ratio of the all metabolites to $\mathrm{tCr}$ for Group 1 and 2.

\section{Discussion}

In the current clinical approach, there is no established diagnostic algorithm in

Table 1. Ratios of the all metabolites to $\mathrm{tCr}$ for Group 1 and 2 and $\mathrm{P}$ values.

\begin{tabular}{cccccc}
\hline & \multicolumn{3}{c}{ Group 1 } & \multirow{2}{c}{ Group 2 } \\
\cline { 2 - 5 } & $($ Mean $\pm \mathrm{SD})$ & (Median) & $($ Mean $\pm \mathrm{SD})$ & (Median) & \\
\hline GABA/tCR & $0.589 \pm 0.194$ & 0.515 & $0.230 \pm 0.067$ & 0.220 & $\mathbf{0 . 0 0 2}$ \\
Ins/tCR & $0.610 \pm 0.107$ & 0.610 & $0.555 \pm 0.108$ & 0.535 & 0.330 \\
tNAA/tCR & $1.106 \pm 0.218$ & 1.205 & $1.538 \pm 0.401$ & 1.705 & $\mathbf{0 . 0 2 8 ^ { * }}$ \\
tCho/tCR & $0.314 \pm 0.027$ & 0.310 & $0.342 \pm 0.031$ & 0.330 & 0.091 \\
Glx/tCR & $0.619 \pm 0.223$ & 0.223 & $0.553 \pm 0.173$ & 0.510 & 0.477 \\
\hline
\end{tabular}

${ }^{\star} P<0.05$, significant. ${ }^{*}$ GABA $(\gamma$-Aminobutyric acid), Ins (myo-inositole), tNAA (total N-Acetylaspartate), tCho (total choline), tCr (total creatine) and GLx (glutamine + Glutamat)).

Table 2. Geographical data and metabolite levels of all the participants.

\begin{tabular}{ccccccccc}
\hline Participants & Group & Gender & GABA & Ins & tNAA & tCho & tCr & Glx \\
\hline 1 & 1 & F & 118 & 97.5 & 133 & 37.5 & 136 & 30.9 \\
2 & 1 & F & 80.2 & 66.6 & 89.1 & 32.9 & 112 & 46.8 \\
3 & 1 & F & 158 & 146 & 238 & 69,2 & 192 & 121 \\
4 & 1 & F & 82.5 & 90.9 & 196 & 52.3 & 153 & 130 \\
5 & 1 & F & 55.4 & 115 & 139 & 55.5 & 176 & 93.8 \\
6 & 1 & M & 73.4 & 65.2 & 194 & 45.6 & 151 & 117 \\
7 & 1 & M & 84.3 & 111 & 230 & 56.1 & 175 & 156 \\
8 & 1 & M & 90.3 & 96.4 & 220 & 56.7 & 188 & 119 \\
9 & 2 & F & 21.3 & 43.3 & 154 & 27.9 & 84.7 & 29 \\
10 & 2 & F & 10.5 & 35.4 & 113 & 23 & 69.2 & 51.9 \\
11 & 2 & F & 13.5 & 41 & 129 & 27.6 & 72.6 & 34.9 \\
12 & 2 & M & 21.8 & 28.8 & 134 & 27.2 & 71.5 & 31.8 \\
13 & 2 & M & 24.5 & 55.2 & 101 & 24.3 & 78.4 & 60.6 \\
14 & 2 & M & 24.9 & 90.1 & 117 & 43.9 & 139 & 75.5 \\
\hline
\end{tabular}

${ }^{*}$ M: male; F: female; Group 1: BMS patients; Group 2: healthy controls. ${ }^{* *}$ GABA ( $\gamma$-Aminobutyric acid), Ins (myo-inositole), tNAA (total N-Acetylaspartate), tCho (total choline), tCr (total creatine) and GLx (gluta$\min +$ Glutamat)). 
the diagnosis of BMS. The diagnosis of BMS is commonly made by excluding the other potential diseases; hence, this inevitably leads to a delay in diagnosis and treatment of patients and also causes significant time and financial loss [3]. Furthermore, allergic and systemic diseases those show similar mucocutaneous symptoms and signs further increased diagnostic difficulties in BMS [9].

Whether the central or peripheral nervous system plays a key role in the emergence of BMS has been a debate for many years. Many researchers have focused on the role of peripheral nervous system disorders in the etiology of BMS. Jaakelainen et al. [10] and Forssell et al. [11] claimed that disturbance in chorda tympani related genetic taste pathway plays a critical role in the emergence of BMS. On the other hand, Lauria et al. [12] claimed BMS is not related to a genetic taste pathway but instead is predominantly caused by a trigeminal nerve-mediated neuropathy [12]. Recent work of Puhakka et al. [13] demonstrated loss of nerve damage and loss in tongue biopsy specimen of the BMS patients.

Besides studies that have propounded the role of the peripheral nervous system in the emergence of BMS, a substantial number of authors have emphasized the role of the central nervous system in the emergence of BMS. Hagelberg et al. [14] showed the role of decreased endogenous dopamine levels in etiology of BMS. Galli et al. [15] demonstrated the association between pain perception and psychological situation in the patients. Recent work of López-D'alessandro et al. [5] showed benefits of drugs that increase the level of GABA in BMS, which supported theories that propounds role of central nervous system disorder in the etiology of BMS. Furthermore, the role of benzodiazepines in the treatment of BMS has also been studied very extensively, yet these studies have provided very inconclusive results [16] [17]. Though there has been a debate, many authors agree upon that, among benzodiazepines, only clonazepam has some beneficial effects in the treatment of the disease [16] [17].

In addition to studies addressing the origin of the disease as peripheral or central nervous system, newer approaches have emerged in recent years to support the view that, in most cases, both central and peripheral mechanisms play an active role in the emergence of BMS. Recent work of Gurvits et al. [18] demonstrated coexistent of increased excitability in peripheral nerves in charge of pain perception and inflammation in the central nervous system in BMS patients.

A small number of studies have evaluated BMS patients with advanced neuroimaging methods including functional MRI (fMRI) and MRS, but all of these studies used complex post-processing methods and in mainly for academic purposes rather than clinical aspect. Shinozaki et al. [19] evaluated BMS patients with fMRI, and claimed the role of both central and peripheral nervous system disorder in BMS. Their fMRI findings also support our findings related to the central nervous system component of the BMS [19]. Sindings et al. [20] demonstrated differences in cortical density and volume between BMS patients and healthy controls especially in the anterior and posterior cingulate gyrus, lobules of the cerebellum, insula/frontal operculum, inferior temporal area, primary 
motor cortex, dorsolateral prefrontal cortex. Khan et al. [21] measured gray matter volume (GMV) in BMS patients with voxel-based manometer, evaluated fractional anisotropy (FA) values of white matter with diffusion tensor imaging (DTI), showed functional connectivity of the brain with resting-state functional MRI (rsfMRI) and demonstrated increased GMV and lower FA values with increased connectivity in medial prefrontal cortex.

Despite all of these studies aforementioned above, there is still no consensus whether central or peripheral pathology plays the leading role in the etiology of BMS. However, determination of central or peripheral problem in BMS patients is very crucial to promptly and accurately decide the treatment of choice. The aforementioned radiological studies used complex post-processing algorithms, and in these work, evaluation mainly depends on a comparison of BMS with other groups. In our study, we obtained our data with an MRI examination only taking few minutes, which is followed by simple post-processing. We highlight that our study should be considered as a first step to clarify the presence of central etiology in patients with BMS despite the low number of cases.

In our patient group, there were no signal abnormalities on the conventional MRI; however, we documented elevated GABA levels in addition to decreased NAA levels suggesting neuronal loss [7] [8]. Intriguingly, increased GABA levels in BMS patients were quite contrary to the expectations. Though it is difficult to completely explain our results, we assume that elevated GABA might likely inhibit the mechanism, which prevents an abnormal sensation, thus, playing a substantial role in the pathophysiology of BMS. Contrary to Guarneri's assumptions [16], we documented normal levels of Cho in BMS patients, which should be increased in inflammatory-demyelinating pathologies or in case of cellular destruction [7] [8]. Therefore, we suppose that neither there are prominent inflammatory or demyelinating pathological changes nor cellular destruction is present in the central nervous system of BMS patients. On the other hand, a mild increase in Ins, despite without a significant difference between BMS and control group, suggest the presence of microglial activation in BMS patients [7] [8]. Furthermore, there was a statistical trend toward in Glx increase in BMS patients compared to healthy participants, suggesting an increased neuronal excitability in the CNS of BMS patients. Increased neuronal excitability phenomenon was also demonstrated by the study of Gurvis et al. [18], though they were focused on the peripheral nervous system rather than the CNS. In summary, despite these alterations might seem to be quite complex, they might serve to enhance our understanding of pathophysiological background of the BMS; thus, enabling us to develop better strategies and treatment alternatives [22].

We had several drawbacks in our study. First, we had a small number of patients since BMS is a rare and not well-known entity. Hence, statically analyzes had to be performed with non-parametric methods due to the small study population. We suggest that further studies with a larger study population might more precisely and accurately demonstrate metabolite changes in central nerv- 
ous system in BMS patients. Therefore, changes in major metabolites such as Glx and Ins might show statistical significant changes between BMS patients and healthy controls. Second, SVS is a very sensitive modality to artifacts and it is reproducibility is quite low; however, we tried to surpass these technical limitations by using standard SVS algorithm, test parameters, ROI and high-resolution images.

In conclusion, we demonstrated a significant increase in GABA, mild increase in Glx and Ins; significant decrease in NAA and normal Cho levels in BMS cases, which indicates central nervous system pathology that is closely associated with patient's symptoms. We suggest that aforementioned alterations in the levels of these metabolites might disrupt the burning sense inhibition in the limbic system by causing dysfunctions in the relevant pathways, and might play a primary role in the development of symptoms in BMS patients. However, our findings should be explored in a larger population in future studies. We claim that levels of metabolites might be more accurately and precisely demonstrated with statistical significant differences between BMS patients and healthy controls (increased GABA, mildly increased Glx and Ins, decreased NAA, normal Cho) in the future studies with larger populations. We also highlight that this protocol might be beneficial for evaluation for central component, and so determination of treatment alternatives in BMS patients.

\section{Conflicts of Interest}

The authors declare no conflicts of interest regarding the publication of this paper.

\section{References}

[1] Gerlinger, I. (2012) Burning Sensation in Oral Cavity-Burning Mouth Syndrome in Everyday Medical Practice. Ideggyogyaszati Szemle, 30, 295-301.

[2] Forssell, H., Teerijoki-Oksa, T., Kotiranta, U., et al. (2012) Pain and Pain Behavior in Burning Mouth Syndrome: A Pain Diary Study. Journal of Orofacial Pain, 26, 117-125.

[3] Nasri-Heir, C. (2012) Burning Mouth Syndrome. Alpha Omegan, 105, 76-81.

[4] de Tommaso, M., Lavolpe, V., Di Venere, D., et al. (2011) A Case of Unilateral Burning Mouth Syndrome of Neuropathic Origin. Headache, 51, 441-444. https://doi.org/10.1111/j.1526-4610.2010.01754.x

[5] López-D’alessandro, E. and Escovich, L. (2011) Combination of Alpha Lipoic Acid and Gabapentin, Its Efficacy in the Treatment of Burning Mouth Syndrome: A Randomized, Double-Blind, Placebo Controlled Trial. Medicina Oral, Patologia Oral y Cirugia Bucal, 16, 635-640. https://doi.org/10.4317/medoral.16942

[6] López, V., Alonso, V., Martí, N., et al. (2009) Marked Response of Burning Mouth Syndrome to Pregabalin Treatment. Clinical and Experimental Dermatology, 34, 449-450. https://doi.org/10.1111/j.1365-2230.2009.03493.x

[7] Oz, G., Alger, J.R., Barker, P.B., et al. (2014) Clinical Proton MR Spectroscopy in Central Nervous System Disorders. Radiology, 270, 658-679.

[8] Gujar, S.K., Maheshwari, S., Björkman-Burtscher, I., et al. (2005) Magnetic Reson- 
ance Spectroscopy. Journal of Neuro-Ophthalmology, 25, 217-226. https://doi.org/10.1097/01.wno.0000177307.21081.81

[9] Scardina, G.A., Pisano, T. and Messina, P. (2007) Burning Mouth Syndrome. Recenti Progressi in Medicina, 98, 120-128.

[10] Jaaskelainen, S.K., Forssell, H. and Tenovuo, O. (1997) Abnormalities of the Blink Reflex in Burning Mouth Syndrome. Pain, 73, 455-460. https://doi.org/10.1016/S0304-3959(97)00140-1

[11] Forssell, H., Jaaskelainen, S., Tenovuo, O., et al. (2002) Sensory Dysfunction in Burning Mouth Syndrome. Pain, 99, 41-47. https://doi.org/10.1016/S0304-3959(02)00052-0

[12] Lauria, G., Majorana, A., Borgna, M., et al. (2005) Tregiminal Small-Fiber Sensory Neuropathy Causes Burning Mouth Syndrome. Pain, 115, 332-337. https://doi.org/10.1016/j.pain.2005.03.028

[13] Puhakka, A., Forssell, H., Soinila, S., et al. (2016) Peripheral Nervous System Involvement in Primary Burning Mouth Syndrome-Results of a Pilot Study. Oral Diseases, 22, 338-344. https://doi.org/10.1111/odi.12454

[14] Hagelberg, N., Forssell, H., Rinne, J.O., et al. (2003) Striatal Dopamine D1 and D2 Receptors in Burning Mouth Syndrome. Pain, 101, 149-154. https://doi.org/10.1016/S0304-3959(02)00323-8

[15] Galli, F., Lodi, G., Sardella, A., et al. (2017) Role of Psychological Factors in Burning Mouth Syndrome: A Systematic Review and Meta-Analysis. Cephalalgia, 37, 265-277. https://doi.org/10.1177/0333102416646769

[16] Guarneri, F., Guarneri, C. and Marini, H. (2008) Contribution of Neuroinflammation in Burning Mouth Syndrome: Indications from Benzodiazepine Use. Dermatologic Therapy, 21, 21-24. https://doi.org/10.1111/j.1529-8019.2008.00228.x

[17] McMillan, R., Forssell, H., Buchanan, J.A., et al. (2016) Interventions for Treating Burning Mouth Syndrome. Cochrane Database of Systematic Reviews, 11, CD002779. https://doi.org/10.1002/14651858.CD002779.pub3

[18] Gurvits, G.E. and Tan, A. (2013) Burning Mouth Syndrome. World Journal of Gastroenterology, 19, 665-672. https://doi.org/10.3748/wjg.v19.i5.665

[19] Shinozaki, T., Imamura, Y., Kohashi, R., et al. (2016) Spatial and Temporal Brain Responses to Noxious Heat Thermal Stimuli in Burning Mouth Syndrome. Journal of Dental Research, 95, 1138-1146. https://doi.org/10.1177/0022034516653580

[20] Sinding, C., Gransjøen, A.M., Schlumberger, G., et al. (2016) Grey Matter Changes of the Pain Matrix in Patients with Burning Mouth Syndrome. European Journal of Neuroscience, 43, 997-1005. https://doi.org/10.1111/ejn.13156

[21] Khan, S.A., Keaser, M.L., Meiller, T.F., et al. (2014) Altered Structure and Function in the Hippocampus and Medial Prefrontal Cortex in Patients with Burning Mouth Syndrome. Pain, 155, 1472-1480. https://doi.org/10.1016/j.pain.2014.04.022

[22] Kim, Y., Yoo, T., Han, P., et al. (2018) A Pragmatic Evidence-Based Clinical Management Algorithm for Burning Mouth Syndrome. Journal of Clinical and Experimental Dentistry, 10, e321. https://doi.org/10.4317/jced.54247 